\title{
SYSTEMATIC REVIEW OPEN Transition of children with life-limiting conditions to adult care and healthcare use: a systematic review
}

\author{
Stuart W. Jarvis $\mathbb{1 D}^{1}$, Daniel Roberts ${ }^{2}$, Kate Flemming ${ }^{3}$, Gerry Richardson ${ }^{4}$ and Lorna K. Fraser ${ }^{1}$
}

BACKGROUND: Improved survival has led to increasing numbers of children with life-limiting conditions transitioning to adult healthcare services. There are concerns that transition may lead to a reduction in care quality and increases in emergency care. This review explores evidence for differences in health or social care use post- versus pre-transition to adult services.

METHODS: MEDLINE, EMBASE, CINAHL, PsychINFO and Social Science Citation Index were searched. Studies published in English since 1990 including individuals with any life-limiting condition post- and pre-transition and reporting a health or social care use outcome were included. Data were extracted and quality assessed by one reviewer with $30 \%$ checked by an independent reviewer. RESULTS: Nineteen papers (18 studies) met the inclusion criteria. There was evidence for both increases and decreases (postversus pre-transition) in outpatient attendance, inpatient admissions, inpatient bed days and health service costs; for increases in Emergency Department visits and for decreases in individuals receiving physiotherapy.

CONCLUSIONS: Evidence for changes in healthcare use post- versus pre-transition is mixed and conflicting, although there is evidence for an increase in Emergency Department visits and a reduction in access to physiotherapy. More high-quality research is needed to better link changes in care to the transition.

Pediatric Research (2021) 90:1120-1131; https://doi.org/10.1038/s41390-021-01396-8

\section{IMPACT:}

- Evidence for changes in healthcare use associated with transition to adult services is conflicting.

- Emergency Department visits increase and access to physiotherapy decreases at transition.

- There are marked differences between care patterns in the United States and Canada.

\section{INTRODUCTION}

Life-limiting conditions (LLCs) include conditions that limit lifei.e. cause premature death, such as Duchenne muscular dystrophy, Batten's disease and conditions that threaten life-i.e. may cause early death but may be cured, such as cancer or liver failure. There are increasing numbers of children and young people with LLCs. ${ }^{1,2}$ Although individual conditions are rare, there are many more children and young people with LLCs in the United Kingdom (approx. 85,000 in 2017/18 ${ }^{3}$ ) than with diabetes mellitus (approx. 36,000 in $2018^{4}$ ) and at least 500,000 children living with similar conditions in the United States. ${ }^{5}$

Children with LLCs often receive specialist paediatric care before transitioning to adult services, typically from age 16 to 19 years in the United Kingdom ${ }^{6}$ and planned to be around age 18 years in the United States. 7 There are many differences in delivery of paediatric and adult care, including relationship continuity and condition expertise. ${ }^{6,8}$ In childhood, allied health services, such as physiotherapy, are provided on an ongoing basis in the UK. There may be direct access to a specialist hospital ward when needed without having to first go through primary care or an Emergency Department. Care is often coordinated by the paediatric specialist with parents directly involved in decisions. ${ }^{9}$ Adults may have care coordinated by a primary care practitioner, with little experience of their condition and with whom they may have had little childhood contact. Allied health services are booked in blocks, with possible gaps in provision (there may be no equivalent adult service).$^{10}$ Hospital access may require primary care or Emergency Department referral. ${ }^{11}$ Where the young people have capacity, they are expected to take more responsibility for care and care decisions, reducing parental involvement. ${ }^{9}$

The transition can be poorly defined and care may also continue in paediatric settings. ${ }^{12}$ Primary care transition training can be lacking, despite efforts at improvement. ${ }^{8}$ Transition can appear abrupt ${ }^{6,13}$ and support varies between conditions, but staff continuity, specialist adolescent clinics, good communication and planning are vital. ${ }^{6}$ Guidelines in the United Kingdom and United States highlight the challenges and need for planning. ${ }^{9,14}$ The United Kingdom Chief Medical Officer's report ${ }^{8}$ into child health called for more research on transition for children with long-term conditions.

Systematic reviews have mainly focussed on transition experiences, interventions and biological indicators of disease progression, not healthcare use. ${ }^{15-19}$ Two reviews looked at experiences and found that many young people felt they lacked knowledge about their conditions, felt unprepared and feared that adult providers had similar limitations. ${ }^{15,17}$ One assessed loss to follow-

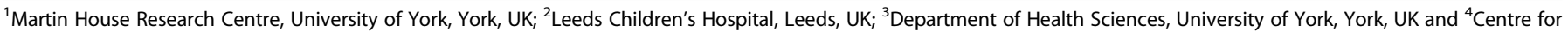
Health Economics, University of York, York, UK

Correspondence: Stuart W. Jarvis (stuart.jarvis@york.ac.uk)

Received: 11 September 2020 Revised: 11 January 2021 Accepted: 14 January 2021

Published online: 2 March 2021 
up and lapse of care, reporting losses of between 7 and $61 \%$ depending on country and definition used. ${ }^{15}$ Other reviews considered healthcare use, but from the perspective of whether there was continuity of care, not quantifying differences in (for example) contacts post- versus pre-transition. ${ }^{16,18}$ These found poor continuity and no standardised transition with, instead, condition-specific programmes. Where biological indicators were assessed, they were commonly compared between intervention and control groups rather than post- versus pre-transition. ${ }^{18,19}$

In short, appraisal of the evidence for changes in healthcare post- versus pre-transition is lacking. Changes are likely to be important to young people and their families. Any increases in (for example) emergency hospital care may have negative implications for them through emotional trauma, disruption and (also, potentially, for health service providers) financial costs. ${ }^{20-25}$

This systematic review aimed to assess evidence for a change in health or social care use for young people with LLC postcompared to pre-transition. This information is needed to identify conditions and areas of care where use varies across the transition, useful for targeting future interventions and research.

\section{METHODS}

The protocol was registered on PROSPERO ${ }^{26}$ (ref. CRD42019156282. ${ }^{27}$ ). Reporting follows the Preferred Reporting Items for Systematic reviews and Meta-Analyses (PRISMA) ${ }^{28}$ and Synthesis Without Meta-Analysis ${ }^{29}$ guidelines.

\section{Eligibility criteria}

Observational studies, randomised controlled trials and studies using quasi-experimental methods (e.g. interrupted time series, regression discontinuity) published in English from 1990 onwards were included if meeting the following criteria (presented in the Population, Exposure, Outcome format). Studies published before 1990 were excluded as widespread survival to adulthood in the LLC population is a relatively recent phenomenon, making older studies less relevant. Systematic reviews were not included, but for any reviews otherwise meeting inclusion criteria, the studies in the review were considered for inclusion.

Population. Children and young people with a LLC, including those treated in both paediatric and adult services (or, if assignment to post- and pre-transition groups was not explicit, including a range of ages spanning at least 15-19 years of age), in Organisation for Economic Co-operation and Development countries. The country limitation avoided comparisons with countries with potentially very different epidemiology and treatment of conditions-e.g. higher prevalence and poorer outcomes for HIV in many developing countries. ${ }^{30}$ Where a study included conditions that may or may not be LLC, depending on severity (e.g. cerebral palsy) only subgroups judged to have a LLC (if provided) were included.

Exposure. The transition from paediatric to adult healthcare services (i.e. in outpatient, inpatient and primary care).

Outcomes. Any measures of health or social care use, excluding those that were purely prescribing (e.g. intravenous antibiotic courses were of interest, oral antibiotic courses were not). Outcomes identified a priori were hospital admissions, bed days, Emergency Department visits, primary care visits and overall costs (direct costs to providers and direct or indirect costs to children and young people and their families).

Assignment to paediatric or adult groups. Study assignments to post- and pre-transition groups were used. It was planned, for any studies that neither assigned individuals to groups nor defined age of transition, to assign the group up to and closest to 16 years as pre-transition and the youngest group aged $\geq 19$ as post- transition, based on common transition ages in the countries studied. ${ }^{6-8}$ However, these rules were not used as all included studies did their own assignment.

\section{Information sources}

MEDLINE, EMBASE, CINAHL, PsychINFO and Social Science Citation Index were searched from 1 January 1990 to 27 April 2020 (date of last search). Forward and reverse citation searching was used for the included studies.

\section{Search strategy}

The search strategy (supplementary material) was developed using the following concepts:

- $\quad$ LLC (using previously developed search strategies ${ }^{31}$ ) AND

- Children/young people (extended from published search strategies $^{14,15,19,32-35}$ ) AND

- Transition (extended from published search strategies $^{14,15,19,32,34}$ )

\section{Study records}

Records were de-duplicated using Endnote ${ }^{36}$ and uploaded to Covidence $^{37}$ for screening.

\section{Selection process}

One reviewer (S.W.J.) screened titles and abstracts against the eligibility criteria. A second reviewer (D.R.) independently screened $20 \%$. Where disagreements occurred, records were retained for full-text screening.

Final selection used full-text records, with two reviewers (S.W.J. and D.R.) assessing all records. A third reviewer (L.K.F.) resolved disagreements.

Data extraction and quality assessment process

One reviewer (S.W.J.) extracted data and assessed quality, with $30 \%$ checked by a fourth reviewer (D.G.-S., see "Acknowledgements"). Disagreements were resolved by discussion. Extracted data items are listed in the extraction form (Supplementary Material). Quality was assessed using a modified Newcastle-Ottawa scale ${ }^{38}$ (Supplementary Material) with one inapplicable question omitted (demonstration that the outcome of interest was not present at the beginning of the study).

\section{Summary measures}

Outcome data were transformed to standardised measures where possible ${ }^{39}:$ mean difference or incidence rate ratio where numbers of outcomes were reported and odds ratios where percentage risk or relative risk was reported. Some reported measures (medians, median differences) were not transformable.

Data synthesis

A meta-analysis was planned, ${ }^{27}$ but studies were insufficiently comparable to undertake this (see "Results"). Alternative approaches were considered: summarising effect estimates ${ }^{40}$ was rejected for similar reasons to conducting a meta-analysis (study designs and measures were too heterogeneous). Instead, $p$ value combination ${ }^{40,41}$ and vote counting ${ }^{40}$ were used. Data were synthesised by outcome as differences by outcome were of interest (i.e. not only whether care use changed but for which outcomes).

$p$ Value combination summarises the strength of evidence for an effect by combining $p$ values reported in studies with a common effect direction. Fisher's $p$ value ${ }^{41}$ was used as a summary measure, indicating whether at least one study showed evidence of an effect. This was visualised using albatross plots, ${ }^{42}$ which plot $p$ value and effect direction against sample size. Visual 
guidelines show the expected plotting position of studies of differing sample sizes with the same effect size-if the true effect size of an intervention was a standardised mean difference of 0.1 , then studies of different sizes would cluster around this guideline. Missing $p$ values were estimated from 95\% confidence intervals $(\mathrm{Cls})^{43}$ or Fisher's exact test. ${ }^{44}$

As not all studies provided $p$ values or data from which these could be estimated, vote counting (counting studies showing effects in each direction, irrespective of statistical significance ${ }^{45}$ ) was also conducted. This was visualised using harvest plots, ${ }^{46}$ bar plots showing the number of studies (number of individual bars) reporting effects in each direction (irrespective of significance) and no effect and their quality scores (height of bar).

Assessment of reporting bias and the strength of the body of evidence

Low numbers of studies with directly comparable effect measures $\left(<10\right.$ for all outcomes ${ }^{47}$ ) prevented the use of funnel plots and associated statistical tests for reporting bias.

Overall strength of evidence was planned ${ }^{27}$ to be assessed using the GRADE framework. ${ }^{48}$ As no overall effect sizes were estimated, this was not done.

\section{RESULTS}

Nineteen papers met inclusion criteria (Table 1) reporting on 18 observational studies-1 study was reported in 2 papers. ${ }^{49,50}$ One systematic review ${ }^{51}$ was also found to be relevant, but its relevant included studies had already been identified in the search and screening process. Figure 1 provides the PRISMA ${ }^{28}$ diagram.

Study characteristics

Settings. Studies took place in six countries: Australia, Canada, France, Germany, United Kingdom, and United States (Table 1), with 12 from North America. Settings ranged from single clinics and hospitals to regional or national analyses. Data sources were mostly routinely collected medical records but included one multiple wave longitudinal cohort study ${ }^{52}$ and one survey. ${ }^{53}$

Conditions studied. Six specific conditions were studied: renal conditions (end-stage kidney disease and kidney transplant recipients), HIV, sickle cell disease, cystic fibrosis, cerebral palsy, and spina bifida. Two studies ${ }^{54,55}$ looked at multiple conditions.

Study designs. Ten studies had longitudinal cohort designs (the same individuals compared post- and pre-transition) and eight were cross-sectional (different individuals compared post- and pretransition). One of the latter ${ }^{56}$ used matching and one was a survey. ${ }^{53}$

Study quality. The observational studies varied in quality, scoring from 2 to 7 of a maximum of 8 on the customised Newcastle-Ottawa scale (Fig. 2). The scale assessed quality of studies for answering the review question-a low score does not necessarily mean that the study was of low quality for its own research question.

Representativeness of study population. Five studies ${ }^{53,57-60}$ used pre-transition groups that were likely not representative of the larger population with the condition (e.g. they used data from a single clinic). The populations included in these studies were likely to reflect the demographics (for example, ethnic group, deprivation level) of individuals geographically close to the clinic andwhere care was provided under private insurance-in possession of appropriate health insurance, rather than the population in the country with the condition. Studies scoring 1 on this criterion used population data (e.g. routine medical records at the nation or state level) or described how participants were representative. All studies drew the post-transition group from the same population as the pre-transition group and so satisfied the second criterion of a representative (of the membership of the pre-transition group) post-transition group.

Ascertainment of transition. The majority of studies used age to allocate individuals to paediatric and adult groups. Nine ${ }^{49,50,52-55,61-63}$ did not provide justification (e.g. that transition invariably happened at that age), so misclassification bias was likely. Seven studies identified transition using clinical records and allowed individuals to transition at different ages.

Controlling for differences between groups. Eight studies ${ }^{55-57,59,60,64-66}$ controlled for age or, where relevant, other differences between groups. Only three $53,56,66$ controlled for severity of condition.

Ascertainment of outcomes and adequacy of follow-up. Most studies used medical records to measure outcomes; the three that did not ${ }^{52,53,67}$ relied on recollection by the young person or service provider. Follow-up was of sufficient length (at least 1 year both post- and pre-transition) in all but two studies, ${ }^{53,55}$ in which minimum follow-up was not stated. Follow-up was inadequate $(>10 \%$ loss without analysis of potential bias) in 8 studies. $^{52,53,55,56,58,60,61,66}$

\section{Outcomes}

Reported outcomes were: outpatient attendances, inpatient admissions, Emergency Department visits, inpatient bed days, intravenous antibiotic courses, physiotherapy, HIV care, General Practice contact, and healthcare costs (Supplemental Table 1).

Data synthesis

A meta-analysis was not conducted as the studies were too heterogeneous in designs (cross-section versus cohort), study populations (North America versus Europe; widely different age ranges in the groups compared) and outcome measures (mean differences, median differences, incidence rate ratios) to provide enough comparable studies for any outcome.

Outpatient attendances. Eleven studies reported outpatient attendance, five showed a lower number post- compared to pre-transition and six showed a higher number (Fig. 3). Quality of studies varied, with Newcastle-Ottawa scores (NOSs) of 3-7. $p$ values were derivable for ten studies. There was strong evidence for both a reduction in at least one study (Fisher's $p<0.001$ ) and an increase (Fisher's $p<0.001$ ). Canadian studies all showed an increase while studies from the United States all showed a decrease. Studies that did not justify assignment to post- and pretransition groups showed larger increases in outpatient attendance than most studies that did justify assignment.

Inpatient admissions. Ten studies looked at inpatient admissions. Six showed a decrease post-transition; four showed an increase (Fig. 3). Studies with results in both directions were of moderateto-high quality (NOS $=4-7$ ). $p$ values were provided or derivable for nine. There was strong evidence for both a reduction (Fisher's $p<0.001$ ) and an increase (Fisher's $p<0.001$ ). Canadian studies all showed a decrease; United States studies showed increases and decreases, but only strong evidence for an increase (Fisher's $p$ values: increase $<0.001$; decrease 0.87 ). Most studies showing a decrease post- versus pre-transition did not justify assignment to the post- and pre-transition groups.

Emergency Department visits. Five studies looked at changes in Emergency Department visits. Three showed an increase, one a decrease $^{60}$ and one no change ${ }^{54}$ (Fig. 3). Studies were of moderate quality (NOS =3-5). $p$ values were derivable for four studies. There was strong evidence for an increase in Emergency Department visits (Fisher's $p<0.001$ ). Two of the three studies showing an increase did not justify assignment to the post- and pre-transition groups. 


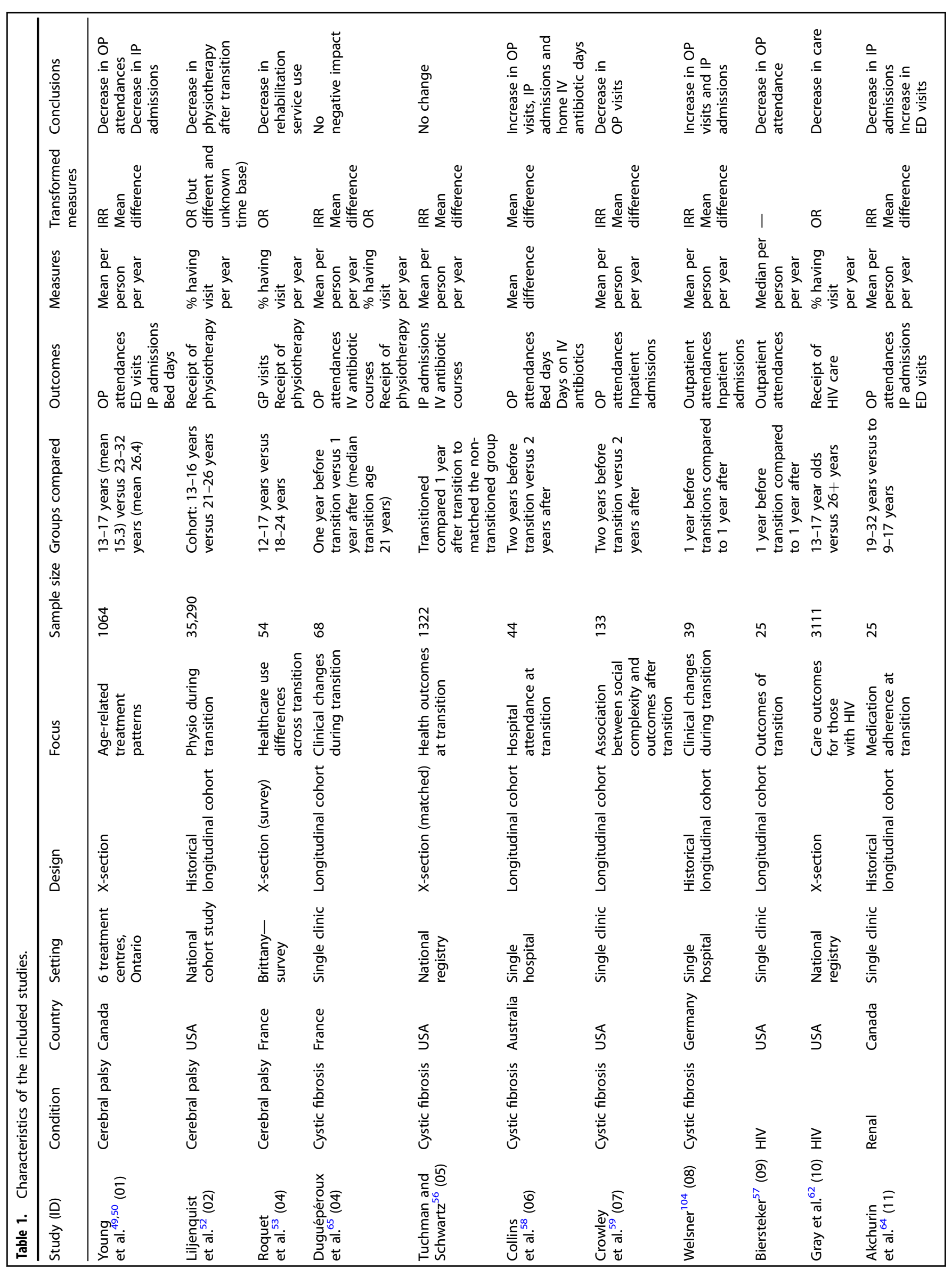




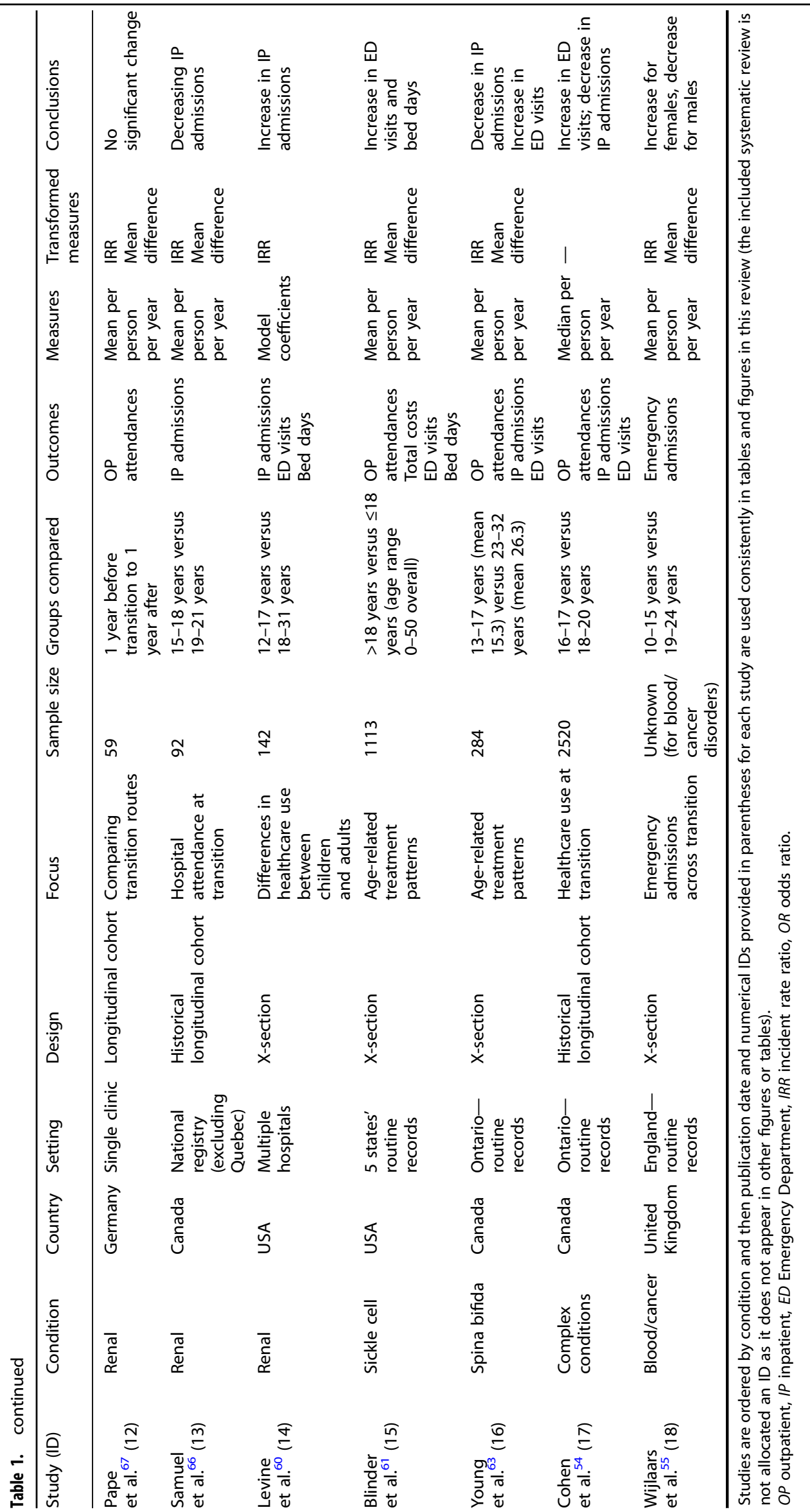




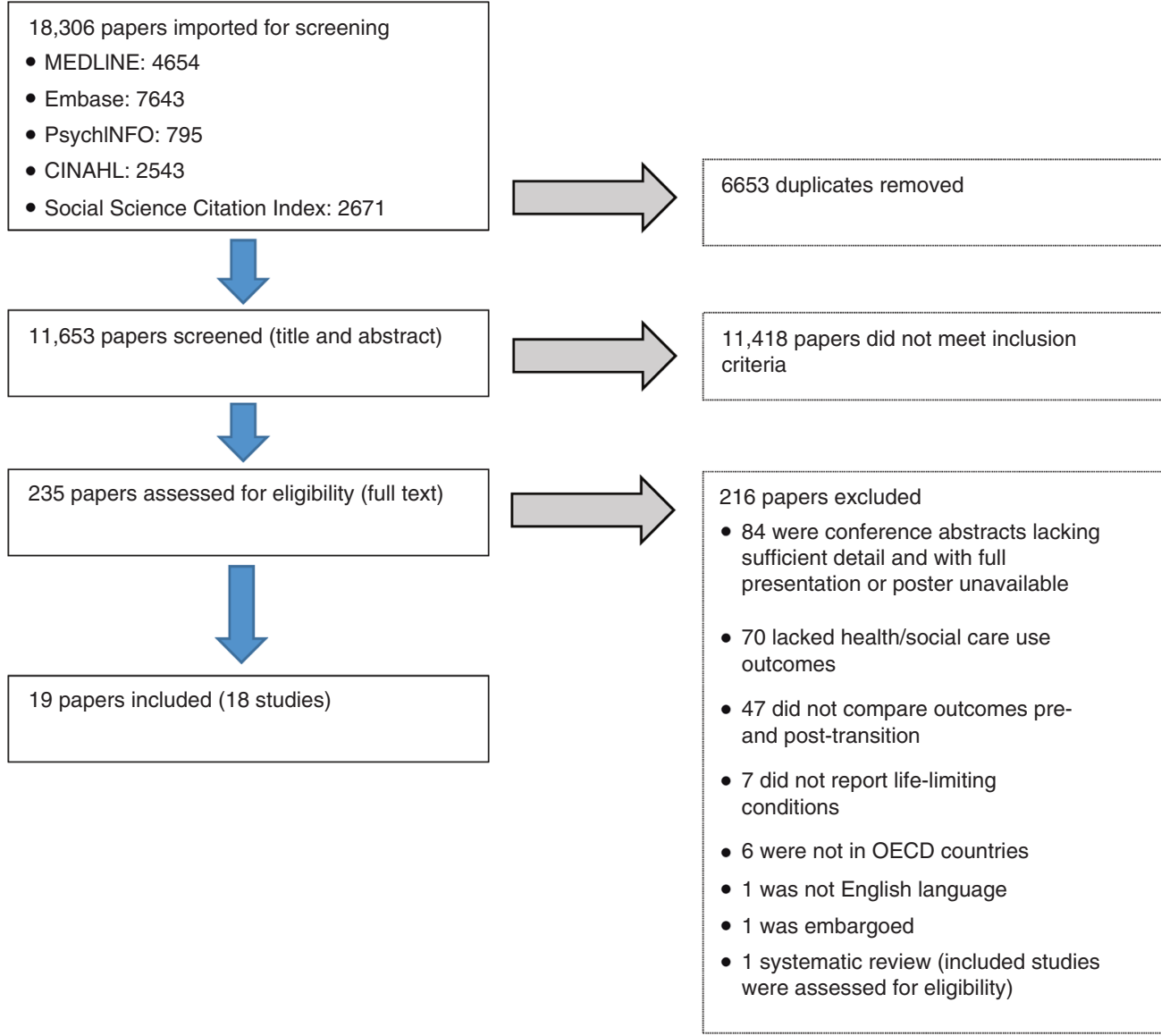

Fig. 1 PRISMA diagram for screening and study selection. Blue arrows show progression of papers between stages and grey arrows show papers rejected at each stage. Numbers are shown from each database and for each reason for rejection at full text eligibility assessment.

Inpatient bed days. Four studies looked at inpatient bed days. Three showed an increase and one a decrease (Fig. 3). Studies were of moderate quality (NOS $=3-5$ ). $p$ values were derivable for three studies and there was evidence for a decrease (Fisher's $p=0.018$ ) and an increase (Fisher's $p=0.002$ ).

Intravenous antibiotic courses. Three studies reported numbers of intravenous antibiotic courses, all showing an increase posttransition (Fig. 3). The studies were of moderate to high quality (NOS $=4-7$ ). $p$ values were derivable in two studies; there was no strong evidence for the observed increases (Fisher's $p=0.103$ ).

Physiotherapy. Three studies reported percentages of individuals in receipt of physiotherapy, with two showing a decrease and one showing no change (Fig. 3). Study quality ranged from low to high (NOS $=2-7$ ). $p$ values were derivable for all studies and there was evidence of a decrease in provision of physiotherapy (Fisher's $p=0.002$ ).

Costs. Only two studies reported differences in total healthcare costs, one showing lower and one higher costs post-transition (Fig. 3). Both studies were of moderate quality (NOS $=3-5$ ). $p$ values were derivable for both. A single study ${ }^{61}$ that splits effects by receipt of iron chelation therapy is shown as two points in the albatross plot but as a single bar in the harvest plot. There was strong evidence of lower (Fisher's $p=0.016$ ) and higher costs (Fisher's $p=0.005$ ).

Other outcomes. One study ${ }^{55}$ reported emergency admissions. This showed a significant increase for females post-transition and a significant decrease for males (Supplemental Table 1). This study was not included in the inpatient admission albatross plot due to a lack of information on sample size and omitted from the harvest plot because it could not be categorised as showing an increase or decrease (having subgroups showing both).

Another study ${ }^{62}$ reported percentages of individuals in receipt of any HIV care and showed a decrease post-transition (Supplemental Table 1).

General Practitioner visits were reported in one study, ${ }^{53}$ which showed that $72 \%$ of individuals visited post-transition and $68 \%$ pretransition (odds ratio $1.2,95 \% \mathrm{Cl} 0.3-4.5$, Supplemental Table 1).

\section{DISCUSSION}

Summary of evidence

The included studies provide conflicting evidence on outpatient attendances, inpatient admissions, inpatient bed days and health service costs (for these outcomes, there was evidence for both increases and decreases post- versus pre-transition). There was greater consistency within countries than between them, where sufficient studies were available to gauge this. There was no evidence for changes in the numbers of intravenous antibiotic courses; there was evidence for both an increase in Emergency Department visits and a reduction in physiotherapy post- versus pre-transition.

Comparisons with other chronic conditions and implications for quality of care

Beyond LLC, for individuals with chronic conditions, there is evidence of disengagement and decreased outpatient attendance after transition. ${ }^{15,32,68}$ This is particularly true for people with diabetes, where an increase in inpatient admissions is also 
Meets criterion

Does not meet criterion

$$
\begin{aligned}
& \text { Does not me } \\
& \text { (1) }
\end{aligned}
$$

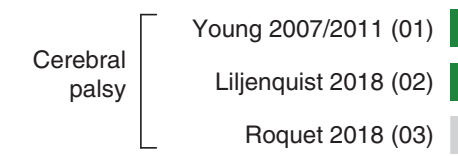

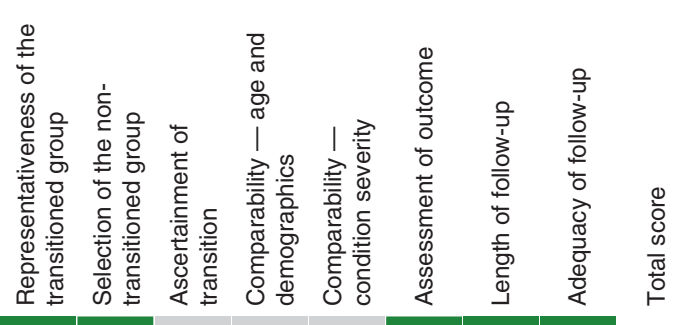

$$
\begin{aligned}
& \text { Cystic }
\end{aligned}
$$

Multiple
conditions
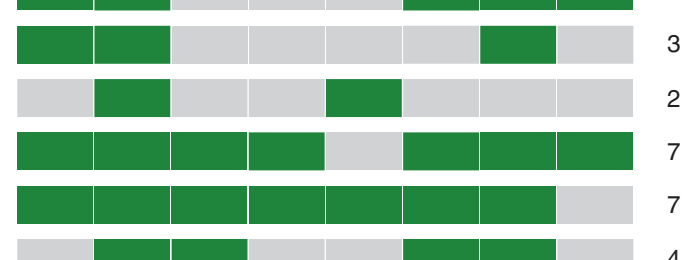

4
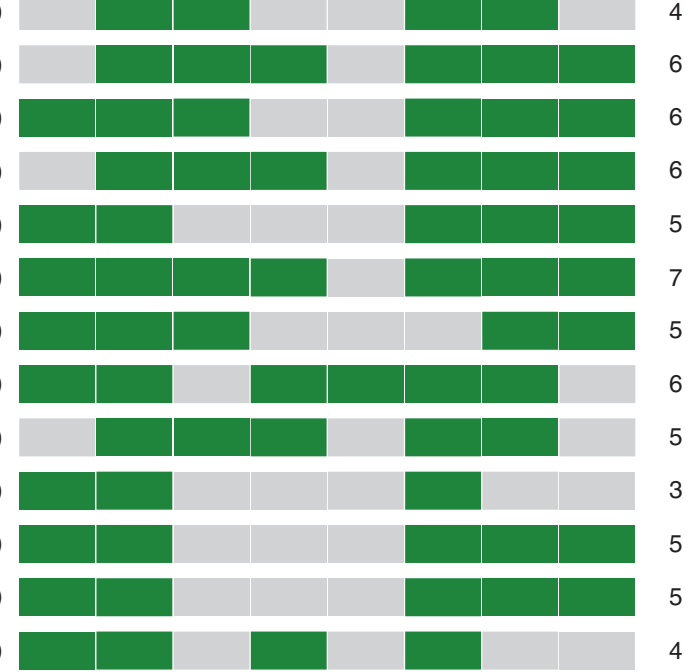

Fig. 2 Quality scores on modified Newcastle-Ottawa scale (see supplement for detailed scoring criteria). Green indicates that a point was scored on each criterion, grey indicates that it was not. Studies, with numerical IDs in parentheses, and conditions studied are indicated to the left and overall scores to the right.

observed, ${ }^{69,70}$ although this varies with continuity of care. ${ }^{71}$ The present review, however, reports a more mixed picture, with evidence emerging for both increased and decreased outpatient attendance. Disengagement may be driven by a perception that further care is unnecessary ${ }^{72}$ and people with milder pre-transition sickle cell disease have lower transition success. ${ }^{73}$ Disengagement may be less likely for people with a LLC due to the severity of their condition necessitating regular contact and due to many young adults with a LLC lacking capacity to decide whether they will or will not attend an appointment, this decision resting instead-as during childhood - with the carer. Implications for care may vary-changes in attendance may reflect different organisation of services postcompared to pre-transition, e.g. paediatrician visits where several different symptoms such as epilepsy and reflux were managed at once may be replaced with multiple outpatient visits to different specialists in adulthood. If either self-care (if appropriate) or primary care partly replace outpatient attendance after transition, the numbers of attendances may decrease. The studies did not report on the levels of attendance at outpatient appointments, which have been shown to be associated with use of emergency hospital care. ${ }^{74}$

The post-transition decrease in inpatient admissions for some included studies was unexpected-it is often anticipated that condition severity (and need for inpatient care) increases with age, independent of transition. However, planned admissions decrease after transition for a range of chronic conditions, ${ }^{75}$ which may explain observed reductions in overall inpatient admissions in some included studies-most reported only overall admissions, not differentiating between emergency and planned. Increasing numbers of adults with chronic conditions are treated in children's hospitals, ${ }^{76}$ which may suggest that suitable care is less available in adult inpatient settings or that transition is delayed for those most in need of inpatient care. An increase in emergency inpatient admissions post-transition may be indicative of worse condition management, but only one study looked at this and reported an increase for women but a decrease for men. ${ }^{55}$

For those with chronic conditions, Emergency Department visits are known to increase at transition ages, ${ }^{77,78}$ and the limited evidence in this review is consistent with this. This may be linked to the transition, e.g. young people seeking hospital rather than primary care post-transition. ${ }^{11,79}$ Alternatively, it may be due to natural progression of condition severity ${ }^{80}$ or increased risk-taking behaviours at the age of transition ${ }^{78}$ (those with long-term conditions are as likely as the those without long-term conditions to increase risk-taking behaviours at this age. ${ }^{81}$ ). Increases in Emergency Department visits are important as adverse care experiences may discourage future adult service engagement. ${ }^{79}$

Inpatient bed days depend on the combination of inpatient admissions and length of stay. Influences on the numbers of admissions discussed above are relevant-there are reasons to both expect more inpatient bed days (due to condition progression and potentially worse outpatient care) and fewer (due to fewer planned admissions ${ }^{75}$ and potentially shorter stays in adult settings. ${ }^{82}$ ) 


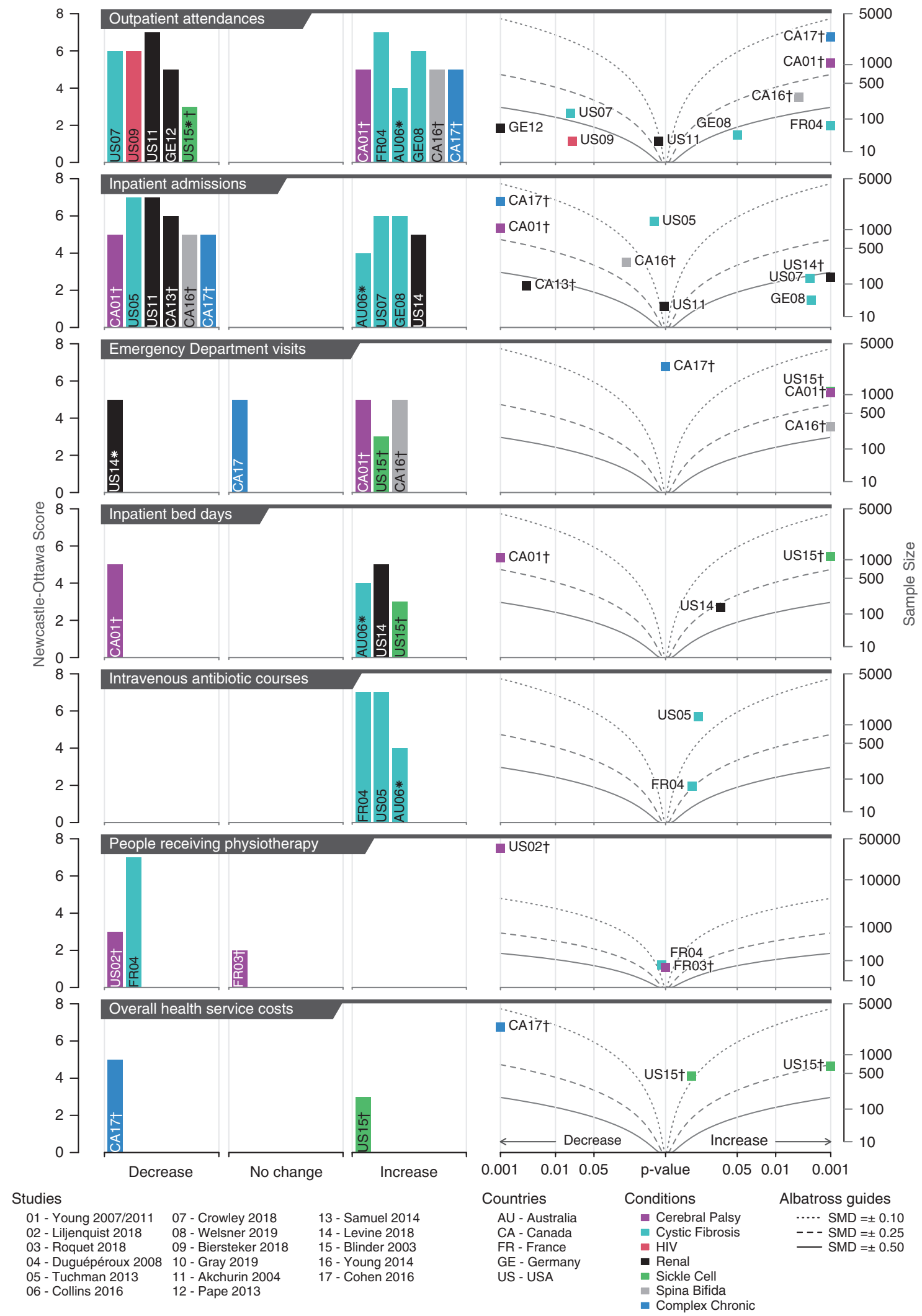

Fig. 3 Harvest plots (left) and albatross plots (right) for the indicated outcomes. Labels, e.g. CA-01, indicate country and numerical study ID. For albatross plots, $p$ values $<0.001$ are plotted at 0.001 . Dagger $(\dagger)$ in the harvest and albatross plots indicates studies that did not provide justification for assignment to post- and pre-transition groups. In the harvest plots, asterisk (*) on a bar indicates a study not included on the corresponding albatross plot (as the $p$ value could not be determined). Curved albatross guidelines are illustrative of the standardised mean difference (SMD) that would give rise to a given $p$ value for a given sample size equally split between post- and pre-transition observation. 
Reduced adherence to medications is observed in late adolescence and with the transition to adult services for many conditions. ${ }^{83-85}$ Antibiotic use is particularly associated with cystic fibrosis to manage infection and is not necessarily indicative of worse outcomes. ${ }^{86,87}$ However, the numbers of intravenous courses of antibiotics increased in the included studies posttransition and one study reported a fall in oral antibiotic use, ${ }^{65}$ which may indicate worse condition management-oral antibiotics are preferred in the first instance. ${ }^{88}$ Two of the included studies showed increases in home administration of antibiotics, ${ }^{56,65}$ which may be appropriate but may indicate a lack of clinical oversight.

Lapses in physiotherapy after transition have long been identified as an issue for children with chronic conditions, ${ }^{14,89}$ so support for this within the included studies is unsurprising. Increases in physiotherapy at home, either alone or with parents, as reported in one study ${ }^{65}$ may be appropriate if sufficient training is given ${ }^{90}$ but may also reflect a lack of service access.

Overall costs may reflect both differences in provider costs and differences in healthcare use. It has been shown that costs for adults admitted to children's hospitals are higher than for those treated in adult hospitals. ${ }^{82}$ Increases may reflect more expensive emergency/hospital care rather than cheaper preventative care in the community or an outpatient setting. ${ }^{91}$ Decreases in costs may indicate more efficient service delivery or reduced access to services. Reflecting this, the evidence on healthcare costs in the included studies was conflicting.

\section{Variations between countries}

Most countries (excepting the United States and Canada) had no more than two studies reporting the same outcome, so no conclusions could be drawn on differences between those countries. Studies from the United States and Canada showed opposite evidence on outpatient attendances (United States: decrease post- versus pre-transition; Canada: increase) and inpatient admissions (United States: increase; Canada: decrease).

Increased outpatient attendances may reflect organisational changes at transition, with multiple adult specialists replacing one paediatric specialist, particularly in Canada, where there are few multi-specialist clinics. ${ }^{92,93}$ It may also reflect differences in funding and access, with Canada having a single-payer system while United States funding is a mix of public and private health insurance. ${ }^{92}$ Insurance type has been associated with different healthcare use for children with a $\operatorname{LLC}^{60}$ and adolescents with special healthcare needs are at higher risk of losing public insurance cover on reaching adulthood ${ }^{94}$ (although insurance type has not been linked with transition success. ${ }^{73}$ ).

Differences in inpatient admissions may be due to differences in disease progression-survival times for cystic fibrosis are greater in Canada than in the United States. ${ }^{95}$ They may also reflect differing demographics-it has been shown that some groups in the US have lower utilisation of outpatient services but higher inpatient use, irrespective of access to healthcare funding. ${ }^{80}$

The few studies looking at inpatient bed days aligned with those for inpatient admissions, with the Canadian study showing a reduction post-transition (despite reporting increased length of stay) ${ }^{49,50}$ and the United States studies showing an increase. ${ }^{60,61}$

Variations between methods of ascertainment of transition Results varied for some outcomes between studies that justified assignment of individuals to the post- or pre-transition groups and studies that did not. Misclassification may explain these differences but would be expected to result in smaller observed changes post- versus pre-transition (due to mixing of the groups) rather than the larger differences or apparent reversal of effect direction observed. Many of the studies not justifying assignment were from Canada and the observed differences largely reflect those between Canada and the United States. Country differences therefore seem more plausible than misclassification bias in explaining the observed variation. One Canadian study ${ }^{54}$ justified assignment by stating that, apart from primary care, transition invariably happened by age 18 years, so other Canadian studies may have assigned post- and pre-transition groups correctly based on this age cut-off, despite not justifying this.

\section{Variations between conditions}

Comparisons between conditions were limited as few conditions had more than two studies reporting the same outcome. Cystic fibrosis studies more commonly reported increases in healthcare use post-transition than decreases-cystic fibrosis has wellestablished transition programmes aimed at maintaining engagement. ${ }^{87}$ The reverse was true for renal conditions, for which studies were largely concerned with paediatric transplant recipients, where many problems have been identified with transition and ongoing engagement in care. ${ }^{96}$

\section{Contribution of this review}

The existing systematic review on healthcare use ${ }^{51}$ had a narrower focus than the present review, including only studies on cystic fibrosis. It found no significant differences in the number of antibiotic therapies and hospital attendance, but an increase in outpatient appointments. The included papers reporting these outcomes were also included in the present study.

The present review is the first to assess evidence across a range of LLCs. It reveals conflicting evidence, some of it patterned by country, suggestive of different healthcare regimes delivering different changes at transition. It finds that assessments of changes in healthcare use at the transition have been inconsistent, with many different outcome measures, designs and methods of ascertaining transition. It highlights the need for more high-quality research in this area.

\section{Limitations}

Included studies. As demonstrated by the NOSs, quality of the included studies varied. Half failed to determine whether individuals were in paediatric or adult care, instead using a simple age cut-off. This was appropriate to the research questions of some studies, but a limitation for the review due to potential misclassification bias.

Most studies failed to separate possible effects of transition, age and condition severity. Only one study ${ }^{56}$ propensity-score matched post and pre-transition groups and two ${ }^{53,66}$ controlled for condition severity. The majority did not control for age, so may have measured effects associated with age and condition progression, rather than transition. There is a need for research that separates associations with age from associations with receipt of either paediatric or adult care.

Differences in age ranges of compared groups limited comparability between studies and were one reason a meta-analysis was not conducted. This reflected differences in the age at which transition takes place, both between and within countries. ${ }^{15,18,51}$ Many studies used means and standard deviations to summarise event counts, even though these were likely to be highly skewed, following a Poisson or negative-binomial distribution. Outcomes measured varied greatly, as observed in reviews of transition for chronic conditions, ${ }^{18,51,97}$ limiting comparability.

Finally, the included studies were concentrated on only a small number of the more common LLCs. In particular, neurological conditions, affecting $12 \%$ of children with a $\operatorname{LLC}^{1}$ and one of the largest groups referred for paediatric palliative care, ${ }^{98,99}$ were under-represented. The generalisability of findings across LLCS as a whole is therefore questionable.

Review limitations. The study limitations prevented a metaanalysis or other effect-size pooling. The synthesis used was appropriate ${ }^{29}$ but explored directions of effect and 
strength of evidence; no conclusions could be drawn on effect size.

There is no definitive list of LLCs and whether a condition is included can depend on severity (e.g. cerebral palsy). Study inclusion in this review depended on the authors' judgement, based on previous work developing lists of LLCs. ${ }^{1}$ Other reviewers might come to different decisions on some studies. Presentation of the results categorised by condition does, however, aid the reader in gauging what effect excluding, for example, cerebral palsy or sickle cell disease would have.

Transition is not always clearly defined, even at the individual level, with some adults continuing to receive some care in paediatric settings. However, the transition is experienced as a very real phenomenon by young people and their families. $6,9,10,13$ Definitions used in many of the studies were based on welldefined cut-offs, such as transfer from a paediatric to an adult clinic.

The search strategy for this review extended previously developed search strategies and was designed to maximise sensitivity. Although initial title and abstract screening was done by one reviewer (with 20\% checked by a second reviewer), full-text review was carried out by two reviewers independently. There is a risk of missed relevant studies among the $80 \%$ of titles and abstracts screened by only one reviewer, ${ }^{100,101}$ but in the $20 \%$ of titles and abstracts that were screened by a second reviewer, no studies rejected by the first reviewer were ultimately included in the review.

Eighty-four conference abstracts were excluded due to the full presentation or poster being unavailable. One study (a doctoral thesis ${ }^{102}$ ) was excluded as the full text was embargoed. It is possible that inclusion of these, had full-text been available, would have altered conclusions.

\section{Future research}

There has been widespread recognition of the need for improvement of the transition and suggestions of how his might be done. $^{18,19,91,103}$ However, more high-quality research is needed that analyses healthcare use across the full range of LLCs to better target interventions with potential to be cost-effective. In particular, the present research in this area is lacking in the following areas:

- possible misclassification bias due to poor ascertainment of transition;

- inconsistent and sometimes inappropriate outcome measurements, e.g. the use of mean values to describe highly skewed distributions;

- a lack of control for potential confounders, particularly increasing severity of condition with increasing age, irrespective of transition status.

Research is needed that more directly links transition and healthcare use, through analysis of the time periods immediately before and after transition and the use of methods enabling causal inference.

\section{CONCLUSIONS}

The evidence on changes in healthcare use post- versus pretransition from paediatric to adult healthcare is mixed and conflicting. There is some evidence of an increase in Emergency Department visits and a reduction in access to physiotherapy but different patterns were identified for outpatient attendances and inpatient admissions between the United States and Canada. This may be linked to differences in organisation and funding of healthcare services, and in at least some populations, significant changes in healthcare use were observed at the transition.

More high-quality research is needed to provide a stronger evidence base for the extent to which widely reported concerns about the transition are reflected in changes in healthcare use and resource utilisation.

\section{ACKNOWLEDGEMENTS}

Dr. Su Golder (Department of Health Sciences, University of York) advised on search strategy development and Dr. Deborah Gibson-Smith (Martin House Research Centre, Department of Health Sciences, University of York) acted as second data extractor and quality assessor. S.W.J. is funded by a National Institute for Health Research Doctoral Research Fellowship (award DRF-2018-11-ST2-013) for this research project. L.K.F. is funded by a National Institute for Health Research Career Development Fellowship (award: CDF-2018-11-ST2-002) for this research project. This publication presents independent research funded by the National Institute for Health Research. The views expressed are those of the authors and not necessarily those of the NHS, the National Institute for Health Research or the Department of Health and Social Care.

\section{AUTHOR CONTRIBUTIONS}

S.W.J. conceptualised and designed the study, designed and conducted the searches and screening, extracted data, reviewed study quality, drafted the initial manuscript, reviewed and revised the manuscript and gave final approval of the version to be published. D.R. contributed to the design of the study, conducted the screening, reviewed and revised the manuscript and gave final approval of the version to be published. K.F. contributed to the design of the study, the design of the searches and choice of databases searched, reviewed and revised the manuscript and gave final approval of the version to be published. G.R. helped conceptualise the study, contributed to the design of the study, reviewed and revised the manuscript and gave final approval of the version to be published. L.K.F. helped conceptualise the study, contributed to the design of the study, the design of searches and choice of databases searched, screening, reviewed and revised the manuscript and gave final approval of the version to be published.

\section{ADDITIONAL INFORMATION}

Supplementary information The online version contains supplementary material available at https://doi.org/10.1038/s41390-021-01396-8.

Competing interests: The authors declare no competing interests.

Consent statement: Patient consent was not required for this study.

Publisher's note Springer Nature remains neutral with regard to jurisdictional claims in published maps and institutional affiliations.

\section{REFERENCES}

1. Fraser, L. K. et al. Rising national prevalence of life-limiting conditions in children in England. Pediatrics 129, e923-e929 (2012).

2. Fraser, L. K. et al. Children in Scotland Requiring Palliative Care: Identifying Numbers and Needs (The ChiSP Study) (University of York, 2015).

3. Fraser, L. K., Gibson-Smith, D., Jarvis, S., Norman, P. \& Parslow, R. C. 'Make Every Child Count' Estimating current and future prevalence of children and young people with life-limiting conditions in the United Kingdom. https://www. togetherforshortlives.org.uk/resource/make-every-child-count/ (2020).

4. Diabetes UK. Us, diabetes and a lot of facts and stats. Diabetes UK: 2019-2002. https://www.diabetes.org.uk/resources-s3/2019-02/1362B_Facts\%20and\% 20stats\%20Update\%20Jan\%202019 LOW\%20RES EXTERNAL.pdf (2019).

5. Himelstein, B. P., Hilden, J. M., Boldt, A. M. \& Weissman, D. Pediatric palliative care. N. Engl. J. Med. 350, 1752-1762 (2004).

6. Care Quality Commission. From the Pond into the Sea. Children's Transition to Adult Health Services (Care Quality Commission, 2014).

7. White, P. H. \& Cooley, W. C. Supporting the health care transition from adolescence to adulthood in the medical home. Pediatrics 142, e20182587 (2018).

8. Lemer, C. Annual Report of the Chief Medical Officer 2012: Our Children Deserve Better: Prevention Pays (Department of Health and Social Care, 2013).

9. American Academy of Pediatrics et al. Supporting the health care transition from adolescence to adulthood in the medical home. Pediatrics 128:182-200 (2011).

10. Kirk, S. \& Fraser, C. Hospice support and the transition to adult services and adulthood for young people with life-limiting conditions and their families: a qualitative study. Palliat. Med. 28, 342-352 (2014). 
11. Hudson, S. M. et al. Factors influencing hospital admissions and emergency department visits among children with complex chronic conditions: a qualitative study of parents' and providers' perspectives. Issues Compr. Pediatr. Nurs. 37, 61-80 (2014).

12. Goodman, D. M. et al. Adults with chronic health conditions originating in childhood: inpatient experience in children's hospitals. Pediatrics 128, 5-13 (2011).

13. Lugasi, T., Achille, M. \& Stevenson, M. Patients' perspective on factors that facilitate transition from child-centered to adult-centered health care: a theory integrated metasummary of quantitative and qualitative studies. J. Adolesc. Health 48, 429-440 (2011).

14. National Institute for Health and Care Excellence. Transition from Children's to Adults' Services for Young People using Health or Social Care Services (NICE, 2016).

15. Heery, E., Sheehan, A. M., While, A. E. \& Coyne, I. Experiences and outcomes of transition from pediatric to adult health care services for young people with congenital heart disease: a systematic review. Congenit. Heart Dis. 10, 413-427 (2015).

16. Doug, M. et al. Transition to adult services for children and young people with palliative care needs: a systematic review. Arch. Dis. Child. 96, 78-84 (2011).

17. Fegran, L., Hall, E. O. C., Uhrenfeldt, L., Aagaard, H. \& Ludvigsen, M. S. Adolescents' and young adults' transition experiences when transferring from paediatric to adult care: a qualitative metasynthesis. Int. J. Nurs. Stud. 51, 123-135 (2014).

18. Crowley, R., Wolfe, I., Lock, K., \& McKee, M. Improving the transition between paediatric and adult healthcare: a systematic review. Arch. Dis. Child. 96, 548-553 (2011).

19. Prior, M., McManus, M., White, P. \& Davidson, L. Measuring the "triple aim" in transition care: a systematic review. Pediatrics 134, e1648-e1661 (2014).

20. Compas, B. E., Jaser, S. S., Dunn, M. J. \& Rodriguez, E. M. Coping with chronic illness in childhood and adolescence. Annu. Rev. Clin. Psychol. 8, 455-480 (2012).

21. Findlay, S., Pinzon, J., Goldberg, E. \& Frappier, J.-Y. Issues of care for hospitalized youth. Paediatr. Child Health 13, 61-64 (2008)

22. Franck, L. S. et al. Predictors of parent post-traumatic stress symptoms after child hospitalization on general pediatric wards: a prospective cohort study. Int. J. Nurs. Stud. 52, 10-21 (2015).

23. Commodari, E. Children staying in hospital: a research on psychological stress of caregivers. Ital. J. Pediatrics 36, 40-40 (2010).

24. DiFazio, R. L. \& Vessey, J. A. Non-medical out-of-pocket expenses incurred by families during their child's hospitalization. J. Child Health Care 17, 230-241 (2013).

25. Wray, J., Lee, K., Dearmun, N. \& Franck, L. Parental anxiety and stress during children's hospitalisation: the StayClose study. J. Child Health Care 15, 163-174 (2011).

26. Booth, A. et al. The nuts and bolts of PROSPERO: an international prospective register of systematic reviews. Syst. Rev. 1, 2 (2012).

27. Jarvis, S. Is there a change in healthcare use at the transition from paediatric to adult services for young people with life-limiting conditions? A protocol for a systematic review. PROSPERO 2019 CRD42019156282. Available from: https:// www.crd.york.ac.uk/prospero/display_record.php? (Accessed 25 Feb 2021).

28. Moher, D., Liberati, A., Tetzlaff, J. \& Altman, D. G. Preferred reporting items for systematic reviews and meta-analyses: the PRISMA statement. Ann. Intern. Med. 151, 264-269 (2009)

29. Campbell, M. et al. Synthesis without meta-analysis (SWiM) in systematic reviews: reporting guideline. BMJ 368, 16890 (2020).

30. Embree, J. The impact of HIV/AIDS on children in developing countries. Paediatr. Child Health 10, 261-263 (2005).

31. Arber, M. Literature Searches for Rapid Review of Care and Support Needs of Children with a Life-Limiting Condition and Their Families - Search Report (York Health Economics Consortium, 2014).

32. Rachas, A. et al. Evaluating continuity during transfer to adult care: a systematic review. Pediatrics 138, e20160256 (2016).

33. Leclercq, E., Leeflang, M. M. G., van Dalen, E. C. \& Kremer, L. C. M. Validation of search filters for identifying pediatric studies in PubMed. J. Pediatrics 162, 629-634.e622 (2013)

34. McPheeters, M. et al. Transition care for children with special health needs. Pediatrics 134, 900-908 (2014).

35. Canadian Health Libraries Association. Association pour l'Avancement des Sciences et des Techniques de la Documentation. Adolescents et Jeunes Adultes (Canadian Health Libraries Association). https://extranet.santecom.qc.ca/wiki/! biblio3s/doku.php?id=concepts:adolescents-et-jeunes-adultes (2018). (Accessed 25 Feb 2021)

36. Clarivate Analytics. Clarivate Analytics Endnote X9. (2021).

37. Covidence. Covidence systematic review software. Veritas Health Innovation Melbourne (2019).

38. Wells, G. et al. Newcastle-Ottawa quality assessment scale cohort studies http:// www.ohri.ca/programs/clinical_epidemiology/oxford.asp (2014). (Accessed 25 Feb 2021).

39. Higgins, J. P., Li, T. \& Deeks, J. J. In Cochrane Handbook for Systematic Reviews of Interventions (eds Higgins, J. P. T. et al.) 143-176 (Wiley, 2019).
40. McKenzie, J. E. \& Brennan, S. E. In Cochrane Handbook for Systematic Reviews of Interventions (eds Higgins, J. P. T. et al.) 321-347 (Wiley, 2019).

41. Becker, B. J. in The Handbook of Research Synthesis (eds Cooper, H. \& Hedges, L. V.) 215-230 (Sage, 1994)

42. Harrison, S., Jones, H. E., Martin, R. M., Lewis, S. J. \& Higgins, J. P. T. The albatross plot: a novel graphical tool for presenting results of diversely reported studies in a systematic review. Res. Synth. Methods 8, 281-289 (2017).

43. Altman, D. G. \& Bland, J. M. How to obtain the $P$ value from a confidence interval. BMJ 343, d2304 (2011).

44. Agresti, A. Categorical Data Analysis (Wiley, 2003).

45. Higgins, J. P. T. \& Wells, G. A. Cochrane Handbook for Systematic Reviews of Interventions (Wiley, 2011).

46. Ogilvie, D. et al. The harvest plot: a method for synthesising evidence about the differential effects of interventions. BMC Med. Res. Methodol. 8, 8 (2008).

47. Page, M. J., Higgins, J. P. T. \& Sterne, J. A. C. in Cochrane Handbook for Systematic Reviews of Interventions (eds Higgins, J. P. T. et al.) 349-374 (Wiley, 2019).

48. Guyatt, G. H. et al. GRADE: an emerging consensus on rating quality of evidence and strength of recommendations. BMJ 336, 924-926 (2008).

49. Young, N. L. et al. Youth and young adults with cerebral palsy: their use of physician and hospital services. Arch. Phys. Med. Rehabil. 88, 696-702 (2007).

50. Young, N. L. et al. Reasons for hospital admissions among youth and young adults with cerebral palsy. Arch. Phys. Med. Rehabil. 92, 46-50 (2011).

51. Coyne, I., Sheehan, A. M., Heery, E. \& While, A. E. Improving transition to adult healthcare for young people with cystic fibrosis: a systematic review. J. Child Health Care 21, 312-330 (2017).

52. Liljenquist, K., O'Neil, M. E. \& Bjornson, K. F. Utilization of physical therapy services during transition for young people with cerebral palsy: a call for improved care into adulthood. Phys. Ther. 98, 796-803 (2018).

53. Roquet, $M$. et al. From childhood to adulthood: health care use in individuals with cerebral palsy. Dev. Med. Child Neurol. 60, 1271-1277 (2018).

54. Cohen, E. et al. Health care use during transfer to adult care among youth with chronic conditions. Pediatrics 137, e20152734 (2016).

55. Wijlaars, L. P. M. M., Hardelid, P., Guttmann, A. \& Gilbert, R. Emergency admissions and long-term conditions during transition from paediatric to adult care: a cross-sectional study using Hospital Episode Statistics data. BMJ Open 8, e021015 (2018).

56. Tuchman, L. \& Schwartz, M. Health outcomes associated with transition from pediatric to adult cystic fibrosis care. Pediatrics 132, 847-853 (2013).

57. Biersteker, S. Outcomes of Transition to Adult HIV Care in Perinatally HIV-Infected Young Adults. FIU electronic dissertation and thesis, Florida International Univ. (2018).

58. Collins, R. et al. The effect of transition from a paediatric to adult cystic fibrosis (CF) centre on clinical status and hospital attendance. Eur. Respir. J. 48, PA671 (2016).

59. Crowley, E. M., Bosslet, G. T., Khan, B., Ciccarelli, M. \& Brown, C. D. Impact of social complexity on outcomes in cystic fibrosis after transfer to adult care. Pediatr. Pulmonol. 53, 735-740 (2018).

60. Levine, R. et al. Disparities in health literacy and healthcare utilization among adolescents and young adults with chronic or end-stage kidney disease. J. Pediatr. Nurs. 38, 57-61 (2018).

61. Blinder, M. A. et al. Age-related treatment patterns in sickle cell disease patients and the associated sickle cell complications and healthcare costs. Pediatr. Blood Cancer 60, 828-835 (2013).

62. Gray, K. M., Wang, X., Li, J. \& Nesheim, S. R. Characteristics and care outcomes among persons living with perinatally acquired HIV infection in the United States, 2015. J. Acquired Immune Defic. Syndr. 82, 17-23 (2019).

63. Young, N. L., Anselmo, L. A., Burke, T. A., McCormick, A. \& Mukherjee, S. Youth and young adults with spina bifida: their utilization of physician and hospital services. Arch. Phys. Med. Rehabil. 95, 466-471 (2014).

64. Akchurin, O. M. et al. Medication adherence in the transition of adolescent kidney transplant recipients to the adult care. Pediatr. Transplant. 18, 538-548 (2014).

65. Duguépéroux, I. et al. Clinical changes of patients with cystic fibrosis during transition from pediatric to adult care. J. Adolesc. Health 43, 459-465 (2008).

66. Samuel, S. M. et al. Avoidable hospitalizations in youth with kidney failure after transfer to or with only adult care. Pediatrics 133, e993-e1000 (2014).

67. Pape, L. et al. Different models of transition to adult care after pediatric kidney transplantation: a comparative study. Pediatr. Transplant. 17, 518-524 (2013).

68. Sheehan, A. M., While, A. E. \& Coyne, I. The experiences and impact of transition from child to adult healthcare services for young people with Type 1 diabetes: a systematic review. Diabet. Med. 32, 440-458 (2015).

69. Busse, F. P. et al. Evaluation of patients' opinion and metabolic control after transfer of young adults with type 1 diabetes from a pediatric diabetes clinic to adult care. Horm. Res. 67, 132-138 (2007).

70. Kipps, S. et al. Current methods of transfer of young people with Type 1 diabetes to adult services. Diabet. Med. 19, 649-654 (2002). 
71. Nakhla, M., Daneman, D., To, T., Paradis, G. \& Guttmann, A. Transition to adult care for youths with diabetes mellitus: findings from a Universal Health Care System. Pediatrics 124, e1134-e1141 (2009).

72. Yeung, E., Kay, J., Roosevelt, G. E., Brandon, M. \& Yetman, A. T. Lapse of care as a predictor for morbidity in adults with congenital heart disease. Int. J. Cardiol. 125, 62-65 (2008)

73. Andemariam, B. et al. Identification of risk factors for an unsuccessful transition from pediatric to adult sickle cell disease care. Pediatr. Blood Cancer 61, 697-701 (2014).

74. Jarvis, S., Livingston, J., Childs, A. M. \& Fraser, L. Outpatient appointment nonattendance and unplanned health care for children and young people with neurological conditions: a retrospective cohort study. Dev. Med. Child Neurol. 61, 840-846 (2019).

75. Rachas, A. et al. Excess mortality and hospitalizations in transitional-age youths with a long-term disease: a national population-based cohort study. PLOS ONE 13, e0193729 (2018)

76. Lam, P. Y., Fitzgerald, B. B. \& Sawyer, S. M. Young adults in children's hospitals: why are they there? Med. J. Aust. 182, 381-384 (2005).

77. Liu, G., Pearl, A. M., Kong, L., Leslie, D. L. \& Murray, M. J. A profile on emergency department utilization in adolescents and young adults with autism spectrum disorders. J. Autism Dev. Disord. 47, 347-358 (2017).

78. Finkelstein, Y. et al. Drug misuse in adolescents presenting to the emergency department. Pediatric Emerg. Care 33, 451-456 (2017)

79. Stollon, N. B. et al. Transitioning adolescents and young adults with sickle cell disease from pediatric to adult health care: provider perspectives. J. Pediatr. Hematol. Oncol. 37, 577-583 (2015).

80. Ozturk, O. D. et al. Disparities in health care utilization by race among teenagers and young adults with muscular dystrophy. Med. Care 52, S32-S39 (2014).

81. Sawyer, S. M., Drew, S., Yeo, M. S. \& Britto, M. T. Adolescents with a chronic condition: challenges living, challenges treating. Lancet 369, 1481-1489 (2007).

82. Okumura, M. J., Campbell, A. D., Nasr, S. Z. \& Davis, M. M. Inpatient health care use among adult survivors of chronic childhood illnesses in the United States. Arch. Pediatr. Adolesc. Med. 160, 1054-1060 (2006).

83. Williams, P. L. et al. Predictors of adherence to antiretroviral medications in children and adolescents with HIV infection. Pediatrics 118, e1745 (2006).

84. Annunziato, R. A. et al. Adherence and medical outcomes in pediatric liver transplant recipients who transition to adult services. Pediatr. Transpl. 11, 608-614 (2007).

85. Shemesh, E., Annunziato, R. A., Arnon, R., Miloh, T. \& Kerkar, N. Adherence to medical recommendations and transition to adult services in pediatric transplant recipients. Curr. Opin. Organ Transplant. 15, 288-292 (2010).

86. VandenBranden, S. L. et al. Lung function decline from adolescence to young adulthood in cystic fibrosis. Pediatr. Pulmonol. 47, 135-143 (2012).

87. Nazareth, D. \& Walshaw, M. Coming of age in cystic fibrosis - transition from paediatric to adult care. Clin. Med. 13, 482-486 (2013).

88. U. K. Cystic Fibrosis Trust Working Group. Antibiotic Treatment for Cystic Fibrosis (Cystic Fibrosis Trust, 2009).

89. Fiorentino, L., Phillips, D., Walker, A. \& Hall, D. Leaving paediatrics: the experience of service transition for young disabled people and their family carers. Health Soc. Care Community 6, 260-270 (1998).

90. Williams, B., Mukhopadhyay, S., Dowell, J. \& Coyle, J. From child to adult: An exploration of shifting family roles and responsibilities in managing physiotherapy for cystic fibrosis. Soc. Sci. Med. 65, 2135-2146 (2007).

91. Vaks, Y. et al. Better health, less spending: redesigning the transition from pediatric to adult healthcare for youth with chronic illness. Healthcare 4, 57-68 (2016).
92. Mossialos, E., Wenzl, M., Osborn, R. \& Sarnak, D. 2015 International Profiles of Health Care Systems (Canadian Agency for Drugs and Technologies in Health, 2016).

93. Kaufman, M. \& Pinzon, J. Transition to adult care for youth with special health care needs. Paediatr. Child Health 12, 785-793 (2007).

94. Okumura, M. J., McPheeters, M. L. \& Davis, M. M. State and national estimates of insurance coverage and health care utilization for adolescents with chronic conditions from the National Survey of Children's Health, 2003. J. Adolesc. Health 41, 343-349 (2007)

95. Stephenson, A. L. et al. Survival comparison of patients with cystic fibrosis in Canada and the United States: a population-based cohort study. Ann. Intern Med. 166, 537-546 (2017).

96. Dallimore, D. J., Neukirchinger, B. \& Noyes, J. Why is transition between child and adult services a dangerous time for young people with chronic kidney disease? A mixed-method systematic review. PLOS ONE 13, e0201098 (2018).

97. Coyne, B., Hallowell, S. C. \& Thompson, M. Measurable outcomes after transfer from pediatric to adult providers in youth with chronic illness. J. Adolesc. Health 60, 3-16 (2017).

98. Vadeboncoeur, C. M., Splinter, W. M., Rattray, M., Johnston, D. L. \& Coulombe, L. A paediatric palliative care programme in development: trends in referral and location of death. Arch. Dis. Child. 95, 686-689 (2010).

99. Feudtner, C. et al. Pediatric palliative care patients: a prospective multicenter cohort study. Pediatrics 127, 1094-1101 (2011).

100. Stoll, C. R. T. et al. The value of a second reviewer for study selection in systematic reviews. Res. Synth. Methods 10, 539-545 (2019).

101. Waffenschmidt, S., Knelangen, M., Sieben, W., Bühn, S. \& Pieper, D. Single screening versus conventional double screening for study selection in systematic reviews: a methodological systematic review. BMC Med. Res. Methodol. 19, 132 (2019).

102. Hardy, R. System-Level Factors Associated with Healthcare Utilization Among Young Adults with Congenital Heart Disease Transitioning to Adult Cardiac Care Settings Using Social Network Analysis. Thesis, University of Colorado at Denver (2018).

103. Brenner, M. et al. Principles for provision of integrated complex care for children across the acute-community interface in Europe. Lancet Child Adolesc. Health $\mathbf{2}$, 832-838 (2018).

104. Welsner, M. et al. Changes in Clinical Markers During A Short-Term Transfer Program of Adult Cystic Fibrosis Patients from Pediatric to Adult Care. The Open Respir Med J. 13, 11-18 (2019).

Open Access This article is licensed under a Creative Commons Attribution 4.0 International License, which permits use, sharing, adaptation, distribution and reproduction in any medium or format, as long as you give appropriate credit to the original author(s) and the source, provide a link to the Creative Commons license, and indicate if changes were made. The images or other third party material in this article are included in the article's Creative Commons license, unless indicated otherwise in a credit line to the material. If material is not included in the article's Creative Commons license and your intended use is not permitted by statutory regulation or exceeds the permitted use, you will need to obtain permission directly from the copyright holder. To view a copy of this license, visit http://creativecommons org/licenses/by/4.0/.

(c) The Author(s) 2021 